\title{
Light-induced absorption in photorefractive strontium barium niobate
}

\author{
Sergei Orlov, Mordechai Segev, ${ }^{*}$ Amnon Yariv, and Ratnakar R. Neurgaonkar ${ }^{\dagger}$ \\ California Institute of Technology, 128-95, Pasadena, California 91125
}

Received February 10, 1994

\begin{abstract}
$\mathrm{Sr}_{0.59} \mathrm{Ba}_{0.41} \mathrm{Nb}_{2} \mathrm{O}_{6}$ and $\mathrm{Sr}_{0.75} \mathrm{Ba}_{0.25} \mathrm{Nb}_{2} \mathrm{O}_{6}$ exhibit an intensity-dependent absorption in the visible spectral range. We perform pump (green)-probe (red) measurements and find variation as high as $0.45 \mathrm{~cm}^{-1}$ in the absorption coefficient, which depends significantly on the polarization of the probe light beam. Our results indicate a nonexponential dark decay time of the induced absorption and suggest that the trapping potential of the shallow traps varies significantly from one trap to another (multiple isolated shallow traps).
\end{abstract}

Photorefractive (PR) strontium barium niobate (SBN) has been the subject of extensive research in recent years. Large PR nonlinearities and relatively fast response times at low power levels ${ }^{1}$ make this material a preferred candidate for a variety of applications, such as optical data storage. Earlier models of the PR effect ${ }^{2}$ assume that charges are given off and trapped by donor and acceptor levels corresponding to different ionization states (for example, $\mathrm{Fe}^{2+}$ and $\mathrm{Fe}^{3+}$ ) of the same atom and consider only a single level of each type. Numerous phenomena, such as intensity-dependent absorption, ${ }^{3,4}$ the nonlinear dependence of the PR response time on the light intensities, ${ }^{5}$ and the intensity dependence of the PR response, can be understood only in the framework of a more-complicated model ${ }^{4-6}$ that involves multiple donor levels and additional shallow traps.

Intensity-dependent absorption and related phenomena were discovered and investigated extensively in different PR crystals and at different optical light intensities in the range of $1 \mathrm{~mW} / \mathrm{cm}^{2}$ to $10^{8} \mathrm{~W} / \mathrm{cm}^{2}$. A recent study ${ }^{7}$ of this effect examined pulsed recording in nominally undoped and Fe-doped SBN:60. In this Letter we investigate light-induced absorption through pump-probe measurements at relatively low optical power densities in PR SBN:60 and SBN:75.

We employ high-optical-quality $6 \mathrm{~mm} \times 6 \mathrm{~mm} \times$ $6 \mathrm{~mm}$ crystal samples of Cr-doped SBN:60 and Cedoped SBN:75. In our experimental setup (Fig. 1) we used a polarized expanded beam $(\lambda=488 \mathrm{~nm})$ of an argon-ion laser as a pump. We measured the light-induced changes in the absorption coefficient by monitoring the transmitted power of a weak, loosely focused probe beam $(\lambda=633 \mathrm{~nm})$ from a low-power $(\approx 1-\mu \mathrm{W}) \mathrm{He}-\mathrm{Ne}$ laser. The polarization of both beams is controlled independently by appropriate half-wave plates. The incident power of the pump beam is controlled by a variable attenuator and is measured with a power meter in the beam path. The pump beam is expanded by a cylindrical lens followed by a spherical lens, which results in a uniform elliptical spot of an area of $0.1 \mathrm{~cm}^{2}$ inside the crystal. The probe beam occupies a much smaller area and is enclosed entirely by the pump inside the crystal. The SBN sample is placed on a bulk copper plate to minimize potential heating by the intense pump laser light. Our measurements are taken in a single-pulse regime, i.e., the pump beam is blocked by an electromechanical shutter (with a response time of $\sim 50 \mu \mathrm{s}$ ) and is switched on only for short periods of time $(0.2$ to $20 \mathrm{~s})$. The power of the transmitted probe is monitored and recorded by a storage oscilloscope.

The light-induced absorption coefficient $\alpha_{I}$ is defined ${ }^{4}$ by

$$
\frac{I_{S}\left(\text { with } I_{P} \text { on }\right)}{I_{S}\left(\text { with } I_{P} \text { off }\right)}=\exp \left(-\alpha_{I} d\right),
$$

where $I_{S}$ and $I_{P}$ are the probe and the pump intensities at the crystal output, respectively, and $d$ is the length of interaction (crystal thickness). For evaluation of some important material parameters, such as the density of the shallow traps in the crystals and the dark ionization rate, from our experimental data, several significant (though commonly used for ferroelectric PR materials) assumptions need to be made. First, we assume ${ }^{8}$ that all the shallow traps have the same thermal ionization rate $\beta_{2}$. Second, we assume that the density of the free electrons reaches its steady state much faster than does the concentration of charged impurities (adiabatic approximation). Finally, we assume that at low light intensities (i.e., $s_{2} I \ll \beta_{2}$, where $s_{2}$ is the photoionization cross section for occupied shallow traps) most of the shallow

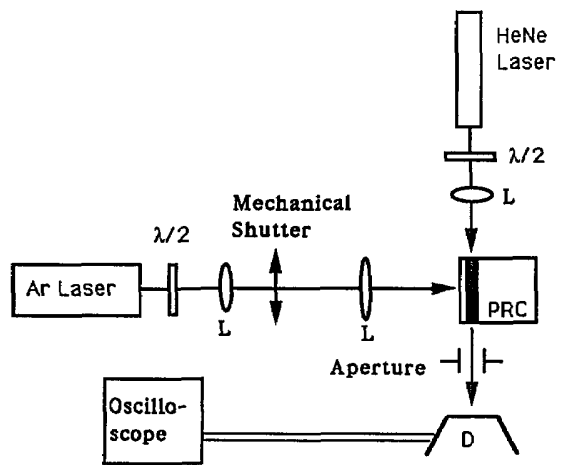

Fig. 1. Experimental setup: L's, lenses; $\lambda / 2$ 's, halfwave plates; PRC, photorefractive crystal; $D$, photodetector. 


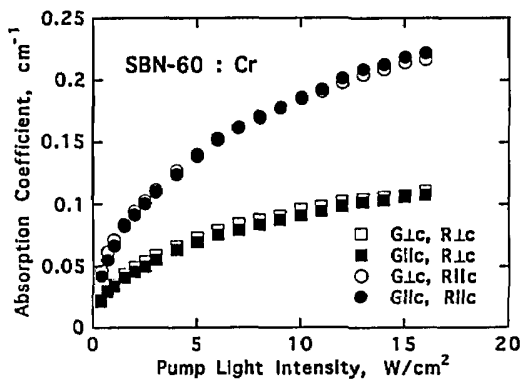

Fig. 2. Light-induced absorption coefficient at $\lambda=$ $633 \mathrm{~nm}$ versus the intensity of the pump beam $(\lambda=488 \mathrm{~nm}$ ) in SBN:60:Cr. $R$ (red) and $G$ (green) denote the polarizations of the probe and the pump, respectively. The linear absorption of the probe beam is $\alpha_{\|}=0.4 \mathrm{~cm}^{-1}$ for extraordinary polarization and $\alpha_{\perp}=0.7 \mathrm{~cm}^{-1}$ for ordinary polarization.

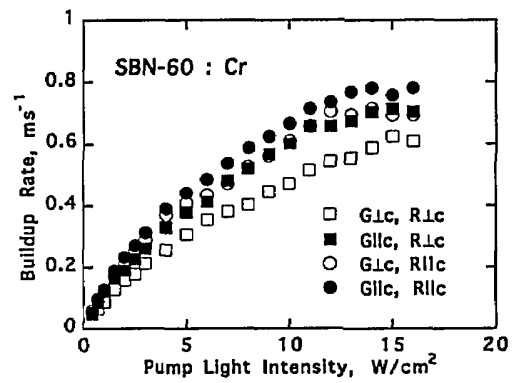

Fig. 3. Buildup rate of the induced absorption at $\lambda=$ $633 \mathrm{~nm}$ versus the intensity of the pump beam $(\lambda=$ $488 \mathrm{~nm}$ ) in SBN:75:Cr.

traps are ionized, their density remains constant, and the density of the free electrons in the conduction band $n$ is dominated primarily by the photoionization and the recombination involving the primary deep PR centers (although $n$ is much smaller than the density of the PR impurities). At intensities far below the saturation of the light-induced absorption effects $\left(s_{2} I \ll \beta_{2}\right)$, the rate at which the population of the shallow traps, and consequently the light-induced absorption coefficient, relaxes to a given equilibrium state is given by ${ }^{9}$

$$
\frac{1}{\tau}=\beta_{2}+s_{2} I+\gamma_{2} n,
$$

where $\gamma_{2}$ is the recombination constant (for electrons) on the shallow traps. Note that at low intensities $n$ is proportional to $I$.

We now measure both the light-induced absorption coefficient $\alpha_{I}$ and its response time $\tau$. First, we investigate the absorption of a weak red $\mathrm{He}-\mathrm{Ne}$ probe induced by a strong green pump in a SBN:60:Cr crystal sample for the different polarizations of both the pump and the probe beams. The steady-state values of $\alpha_{I}$ are shown in Fig. 2. The light-induced absorption coefficient saturates at high pump intensities and appears to be fairly insensitive to the polarization of the pump (488-nm) beam. The dependence of $\alpha_{I}$ on the probe-beam polarization is much more dramatic. The data in Fig. 2 suggest that, for a given pump intensity and polarization, $\alpha_{I}$ (extraordinary) $/ \alpha_{I}$ (ordinary) $=1.95 \pm 0.10$ (see also Ref. 10). A possible explanation of this strong asymmetry with respect to the polarization of the probe beam may be given if we account for the nature of the material defects that act as the shallow traps. If we assume that these traps are primarily structural defects (e.g., vacancies) rather than impurities and that mutual interaction between them is negligible, the traps and the associated wave functions possess a crystal-imparted symmetry, leading to strong polarization effects. Intuitively, one may view the shallow traps as isolated potential wells with their associated wave functions localized differently along the $a$ and $c$ axes. This may result in a larger dipole moment (and, consequently, a larger photoionization cross section) for the light polarized along the $c$ axis. This explanation, if correct, may also provide useful information about the actual atoms whose absence leads to the shallow traps.

Next we investigate the buildup rate of the induced absorption and define it as the inverse of the time required for the signal to reach the $(1-1 / e)$ level of its saturation value. Our results are presented in Fig. 3. The buildup rate curves are significantly nonlinear with respect to the pump intensity with a pronounced saturation at high pump intensities. Notice that for both polarizations of the probe we observe shorter response times for the extraordinarily polarized pump, a result of the higher absorption of the extraordinarily polarized light $\left(\alpha_{\|}=2.0 \mathrm{~cm}^{-1}\right.$ at $\left.\lambda=488 \mathrm{~nm}\right)$ than of the ordinarily polarized one $\left(\alpha_{\perp}=1.5 \mathrm{~cm}^{-1}\right.$ at $\left.\lambda=488 \mathrm{~nm}\right)$. The linear behavior at the low-intensity branch of curves permits [Eq. (2)] an estimate of the effective dark decay rate $\beta_{2}$ of the population of the shallow traps as a result of the thermal ionization: $\beta_{2}=30 \pm 5 \mathrm{~s}^{-1}$. This value is very close to sim-

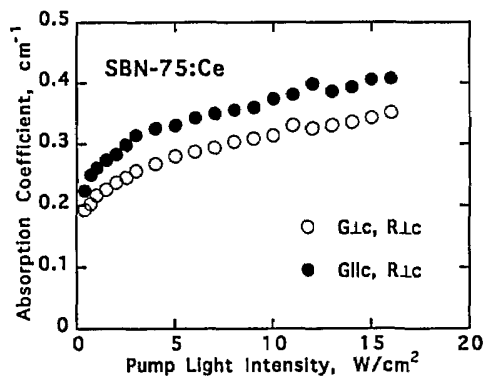

Fig. 4. Light-induced absorption coefficient at $\lambda=$ $633 \mathrm{~nm}$ versus the intensity of the pump beam $(\lambda=$ $488 \mathrm{~nm}$ ) in SBN:75:Ce. The probe beam is ordinarily polarized in both cases, and its linear absorption coefficient is $0.6 \mathrm{~cm}^{-1}$.

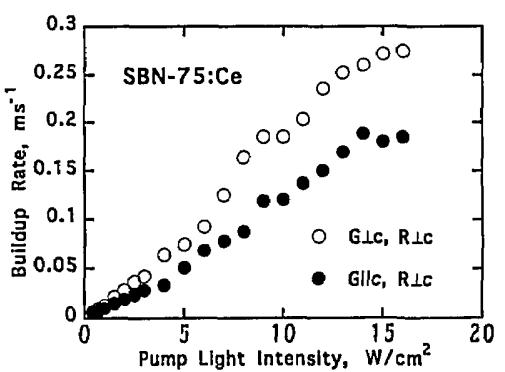

Fig. 5. Buildup rate of the induced absorption at $\lambda=$ $633 \mathrm{~nm}$ versus the intensity of the pump beam $(\lambda=$ $488 \mathrm{~nm}$ ) in SBN:75:Ce. The probe beam is ordinarily polarized in both cases. 


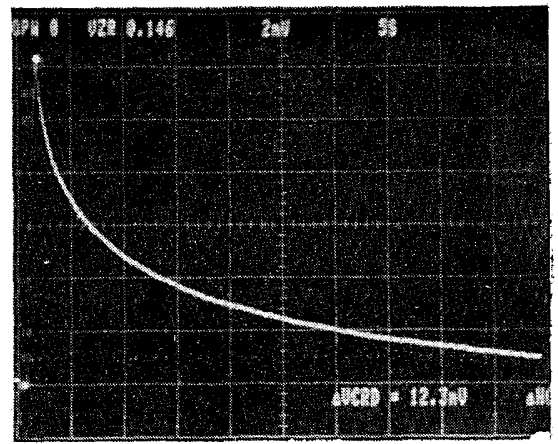

Fig. 6. Nonexponential dark decay of the induced absorption in SBN:75:Ce, after an initial 200-ms pump light pulse. The vertical axis corresponds to the induced absorption $\left(0.025 \mathrm{~cm}^{-1} /\right.$ division $)$, and the horizontal scale is $5 \mathrm{~s}$ /division.

ilar data obtained in the $\mathrm{BaTiO}_{3}$ crystals. ${ }^{4}$ However, even at moderate intensities $\left(>5 \mathrm{~W} / \mathrm{cm}^{2}\right)$ the response time is approximately 1.5 to $2 \mathrm{~ms}$, which is significantly shorter than both the PR and the thermal response times for SBN crystals. ${ }^{11}$ This observation raises the possibility of fast recording of photochromic gratings by light-induced absorption.

We used the same setup to investigate the lightinduced absorption in a Ce-doped SBN:75 sample. In this crystal strong PR coupling is present for extraordinarily polarized light. Therefore the light-induced absorption measurements are performed only with ordinary polarization of the probe beam. Our experimental data, which are shown in Figs. 4 and 5, indicate that even low pump intensities $\left(<5 \mathrm{~W} / \mathrm{cm}^{2}\right)$ induce a large increase in the absorption at the probe wavelength. The linear absorption of the crystal at the pump wavelength $(\lambda=488 \mathrm{~nm})$ is nearly equal for the extraordinary $\left(\alpha_{\|}=4.7 \mathrm{~cm}^{-1}\right)$ and the ordinary $\left(\alpha_{\perp}=4.8 \mathrm{~cm}^{-1}\right)$ polarizations. The pump intensity at which the lightinduced absorption coefficient exhibits saturation appears to be much smaller for SBN:75. This may explain the intensity-dependent diffraction efficiency and intensity-dependent wave coupling observed in some SBN:75 PR crystals. It should be noted that the dark decay (shown in Fig. 6) and the buildup of the light-induced absorption at low light intensities (below saturation, $s_{2} I \ll \beta_{2}$ ) both exhibit a nonexponential temporal behavior. This phenomenon is attributed $^{12}$ to the fact that the trapping potential of the shallow traps may differ significantly from one isolated trap to another (inhomogeneous broadening) rather than having a single energy value. This, in turn, implies that the effective relaxation time depends on the initial distribution of the occupied shallow traps and that the decay toward equilibrium obeys a logarithmic, rather than exponential, law within a certain time window. ${ }^{13}$ Our observations of the dark decay indicate that the relaxation time for the light-induced absorption in SBN:75 may be as long as 3.0 to $5.0 \mathrm{~s}$. This indicates that in this material the secondary traps are relatively deep. However, even at moderate pump intensities $\left(\sim 10 \mathrm{~W} / \mathrm{cm}^{2}\right)$ the buildup of the absorption is very fast $(<5 \mathrm{~ms})$.
Finally, we obtained a lower estimate for the density of the shallow levels for the crystals that we studied by using the absorption cross section evaluated ${ }^{4}$ for $\mathrm{BaTiO}_{3}: s_{2}=5 \times 10^{-18} \mathrm{~cm}^{2}$. The lower limit for the density of the secondary traps $N_{\text {sh }}$ is given by $N_{\text {sh }} \approx \alpha_{I}(\max ) / s_{2}=5 \times 10^{16}$ to $5 \times 10^{17} \mathrm{~cm}^{-3}$. The actual value may be at least $2-10$ times larger, since the approximate formula that we use assumes that all the traps are occupied.

In conclusion, we have presented experimental evidence for light-induced, polarization-dependent absorption in PR SBN:60 and SBN:75. Large changes in the crystal absorption can be induced even at moderate $\mathrm{cw}$ light intensities in the visible range. This fact has a significant effect on applications, such as optical data storage, for which SBN is a potential candidate.

This research was sponsored by the U.S. Army Research Projects Agency.

*Present address, Department of Electrical Engineering, and Advanced Technology Center for Photonics and Optoelectronic Materials and Princeton Material Institute, Princeton University, Princeton, New Jersey 08544.

†Permanent address, Rockwell International Science Center, 1049 Camino dos Rios, Thousand Oaks, California 91360.

\section{References}

1. R. R. Neurgaonkar and W. K. Cory, J. Opt. Soc. Am. B 3, 274 (1986).

2. N. V. Kukhtarev, V. B. Markov, S. G. Odulov, M. S. Soskin, and V. L. Vinetskii, Ferroelectrics 22, 949 (1979).

3. A. Motes and J. J. Kim, J. Opt. Soc. Am. B 4, 1379 (1987).

4. G. A. Brost, R. A. Motes, and J. R. Rotge, J. Opt. Soc. Am. B 5, 1879 (1988).

5. L. Holtmann, Phys. Status Solidi A 113, K89 (1989).

6. D. Mahgerefteh and J. Feinberg, Phys. Rev. Lett. 64, 2195 (1990); P. Tayebati and D. Mahgerefteh, J. Opt. Soc. Am. B 8, 1053 (1991).

7. S. A. Holmstrom, B. Taheri, and R. J. Reeves, in $D i$ gest of Topical Meeting on Photorefractive Materials, Effects and Devices (Optical Society of America, Washington, D.C., 1993), p. 255.

8. This assumption is equivalent to assuming that the shallow traps occupy a single level and is valid for $\mathrm{SBN}: 60$ but, as shown below, is not valid for SBN:75.

9. L. Holtmann, K. Buse, G. Kuper, A. Groll, and E. Krätzig, Appl. Phys. A 53, 81 (1991).

10. After this Letter was submitted, a similar observation that indicated an identical ratio between light-induced absorption coefficients of extraordinarily and ordinarily polarized light in SBN:60:Ce was reported by K. Buse, R. Pankrath, and E. Krätzig, Opt. Lett. 19, 260 (1994).

11. M. Horowitz, R. Daisy, O. Werner, and B. Fisher, Opt. Lett. 17, 475 (1992).

12. H. Mabuchi, E. S. Polzik, and H. J. Kimble, "Bluelight-induced infrared absorption in $\mathrm{KNbO}_{3}$ ” J. Opt. Soc. Am. B (to be published).

13. R. Street and J. C. Wooley, Proc. Phys. Soc. A 62, 562 (1949). 